\title{
The mathematical theory of evidence - A short introduction
}

\author{
Jürg Kohlas \\ Institute of Informatics \\ University of Fribourg, CH 1700 Fribourg, Switzerland. \\ e-mail: Juerg.Kohlas@unifr.ch
}

\begin{abstract}
Evidence or Dempster-Shafer theory is used to model information which is both uncertain and imprecise. Such a piece of information can be captured by the mathematical model of a hint. It is shown how hints can be combined and used to judge hypotheses by degrees of support and plausibility. Applications of this theory to statistical inference, diagnostics and risk analysis, and to decision analysis are discussed. The practical implementation of Dempster-Shafer theory depends on appropriate computational architectures both for modeling and for the inference mechanisms. A fundamental scheme for local computation in hypertrees is presented.
\end{abstract}

\section{ORIGINS}

The term "Evidence Theory" was coined by Glenn Shafer in his book "A Mathematical Theory of Evidence", published by Princeton University Press in 1976. This work was initiated by a course on statistical inference taught by Arthur Dempster at Harvard University. Dempster developed there a theory of lower and upper probabilities in an attempt to reconcile Bayesian statistics with Fisher's fiducial argument (Dempster 1967, 1968). As Shafer states in the preface of his book "... It offers a reinterpretation of Dempster's work, a reinterpretation that identifies his "lower probabilities" as epistemic probabilities or degrees of belief, takes the rule for combining such degrees of belief as fundamental, and abandons the idea that they arise as lower bounds over classes of Bayesian probabilities." The rule mentioned in this statement became known as Dempster's rule.

"Evidence" is an appealing term and it is no surprise that the "Theory of Evidence" found much interest among knowledge engineers who have to model uncertain information. After a lot of ad hoc attempts to model uncertainty in expert systems, a serious theory seemed finally available to treat one of the basic problems of the field. However, as the theory is not really compatible with the simple paradigms of early rule based expert systems, misinterpretations and oversimplistic misuses of the theory caused much

Research partly supported by Grants No. 21-30186.90 and 21-32660.91 of the Swiss National Foundation for Scientific Research and the Swiss Federal Office for Science and Education, Esprit Basis Research Activity Project DRUMS II (Defeasible Reasoning ans Uncertainty Management Systems). 
deception. Only slowly begins the true nature and the real meaning of evidence theory to emerge and to be understood. And this will hopefully be the starting point for fruitful and appropriate applications to the modeling of uncertainty, decision analysis and knowledge engineering in general.

Evidence is a notion which probably can never be fully captured by a single formal theory. In this introduction however "Theory of Evidence" will be understood in a narrow sense as the theory introduced by Dempster and Shafer and variants thereof. This particular theory is also often called Dempster-Shafer theory. It is clear today that this theory can be given various different, but essentially equivalent mathematical forms. Some of them are based on probability theory, others are axiomatic theories, a priori without a reference to probability theory. According to this distinction we classify the approaches broadly into probabilistic approaches and non probabilistic ones. The former try to integrate evidence theory into the framework of classical probability theory, whereas the latter deliberately go beyond classical probability. May be it would be more correct to call them rather non-standard probability theories than non-probabilistic ones. Despite the differences in approach and interpretation, all of them lead essentially to mathematically equivalent theories - at least in the finite case. That is they share the same basic theorems and the same computational procedures apply. In this introduction a probabilistic approach to evidence theory will be given, which is based upon Dempster's original work.

An important pragmatic consideration regarding the usefulness of evidence theory is the question, whether there are indeed important practical applications which can be described in a natural and useful way by the structures provided by the theory of evidence. This question is addressed in section 3. From the point of view of computation, evidence theory is complex in the usual technical sense. Nevertheless there exist methods which greatly improve the efficiency of computations. Also, based on these methods, software packages begin to appear which permit to apply evidence theory to practical problems. This subject is discussed in section 4 .

Today there are not many comprehensive and introductory presentations and surveys of evidence theory. Beside the book of Shafer (1976 a), which is still a recommended reading, there are recent surveys by Smets, 1988, Shafer, 1990 and 1992, Kohlas, Monney, 1994 a. Recent books on uncertainty including chapters on evidence theory are by Kruse et al. (1991) and Hajek et al., 1992. Finally, let's mention the recent book by Fedrizzi et al. (1993) and Kohlas, Monney (1995) devoted entirely to Dempster-Shafer theory.

\section{A MATHEMATICAL MODEL OF HINTS}

Suppose that a certain precise question, whose answer is unknown, has to be studied and the elements $\theta$ of $\Theta$ represent the possible answers to the question. This means that exactly one of the $\theta \in \Theta$ is the correct answer, but it is unknown which one. However there is some information or evidence available relative to this question. This information allows for several, distinct interpretations, depending on some unknown circumstances and these interpretations are represented by the elements $\omega$ of $\Omega$. This means that there is exactly one correct interpretation $\omega$ in $\Omega$, but again it is unknown which one. Not all interpretations are equally likely and the probabilities $p(\omega)$ describe these different likelihoods. If $\omega \in \Omega$ is the correct interpretation, then the unknown answer $\theta$ is known to be in the set $\Gamma(\omega)$. Such a piece of information is called a hint (Kohlas, $1993 \mathrm{~b}$ and 
1995). This model allows to give a specific and clear sense to the further important notions introduced by Dempster (1967).

If $H$ is a subset of $\Theta$, the hypothesis that the unknown answer $\theta$ is in $H$ can be considered. In order to judge the hypothesis $H$ in the light of the hint $(\Omega, P, \Gamma, \Theta)$, we may ask, which of the possible interpretations make $H$ necessarily true. These are all interpretations $\omega$ having a non empty set $\Gamma(\omega)$ which is contained in $H$ because if such an interpretation is the correct one, then $\theta \in \Gamma(\omega)$ and thus necessarily $\theta \in H$. Define

$u(H)=\{\omega \in \Omega: \emptyset \neq \Gamma(\omega) \subseteq H\}$.

In the same spirit, we may ask what are the interpretations which would make $H$ not necessarily true, but at least possible. These interpretations are those in

$v(H)=\{\omega \in \Omega: \Gamma(\omega) \cap H \neq \emptyset\}$

because if $\omega \in v(H)$ is the correct interpretation, then $\theta \in \Gamma(\omega)$ and it is at least possible that $\theta$ is in $H$, although not sure.

Note that $v(\Theta)$ contains all interpretations with $\Gamma(\omega) \neq \emptyset$. Now, because $\Theta$ is supposed to contain the true answer, an interpretation $\omega$ which is not in $v(\Theta)$ is not really a possible interpretation. Thus, the correct interpretation must be in $v(\Theta)$ and this supplementary information allows to pass to the conditional probability $p(\omega \mid v(\Theta))=p(\omega) / P(v(\Theta))$ for the possible interpretations in $v(\Theta)$.

As the correct interpretation is unknown, it is not possible to confirm whether it is in $u(H)$ or $v(H)$, that is whether $H$ is true or only possible. But it is at least possible to compute the probabilities that the correct interpretation is in $u(H)$ or $v(H)$ :

$s p(H)=P(u(H) \mid v(\Theta))=P(u(H)) / P(v(\Theta))$
$p l(H)=P(v(H) \mid v(\Theta))=P(v(H)) / P(v(\Theta))$.

Because the $\omega$ in $u(H)$ can all be regarded as "arguments" in favour of the hypothesis $H$, arguments however, which are not sure to hold, but only more or less probable, $s p(H)$ can be regarded as the probability with which $H$ can be infered or proved from the available information $(\Omega, P, \Gamma, \Theta)$. From this point of view $s p(H)$ can be regarded as the degree of support of the hypothesis $H$ induced by the hint $(\Omega, P, \Gamma, \Theta)$. Similarly, all the interpretations $\omega$ in the set $v(H)$ can be regarded as "arguments" for the possibility of $H$. Then $p l(H)$ can be considered as the degree of possibility or plausibility of $H$.

Considering all possible hypotheses $H \subseteq \Theta, s p$ and $p l$ become functions from the power set of $2^{\Theta}$ to $[0,1]$ and as such they are called support (also belief) and plausibility functions. The following theorem collects a number of fundamental properties of these functions.

Theorem 2.1 If sp and $p l$ are defined by (3) and (4) relative to a hint $(\Omega, P, \Gamma, \Theta)$, then

$s p(\emptyset)=p l(\emptyset)=0 ; s p(\Theta)=p l(\Theta)=1$ 
$s p(H) \leq p l(H)$ for all $H \subseteq \Theta$

$s p(H)=1-p l\left(H^{c}\right), p l(H)=1-s p\left(H^{c}\right)$,

and

$s p(H) \geq \sum\left\{(-1)^{|I|+1} s p\left(\cap_{i \in I} H_{i}\right): \emptyset \neq I \subseteq\{1, \ldots, n\}\right\}$

for all $n \geq 1$ and sets $H, H_{i}$ in $\Theta$ such that $H \supseteq H_{i}$ and

$p l(H) \leq \sum\left\{(-1)^{|I|+1} p l\left(\cup_{i \in I} H_{i}\right): \emptyset \neq I \subseteq\{1, \ldots, n\}\right\}$

for all $n \geq 1$ and sets $H, H_{i}$ in $\Theta$ such that $H \subseteq H_{i}$.

A proof of these properties may be found for example in Shafer (1976 a) or Kohlas, Monney (1995). The inequality (8) says that $s p$ is monotone of order $\infty$ and (9) states that $p l$ is alternating of order $\infty$.

It is possible that one has two or more hints or sources of information $\mathcal{H}_{i}=\left(\Omega_{i}, P_{i}, \Gamma_{i}, \Theta\right)$, $i=1, \ldots, m$ relative to the same question. Then this information has to be combined in order to integrate all the available information. As exactly one interpretation $\omega_{i} \in \Omega_{i}$ is the correct one for every hint, there is exactly one correct combined interpretation $\left(\omega_{1}, \ldots, \omega_{m}\right)$. This combined interpretation restricts the correct answer $\theta$ to the question considered into the subset $\Gamma\left(\omega_{1}, \ldots, \omega_{m}\right)=\Gamma_{1}\left(\omega_{1}\right) \cap \ldots \cap \Gamma_{m}\left(\omega_{m}\right)$ of $\Theta$. Thus $\Omega_{1} \times \ldots \times \Omega_{m}$ is the set of all combined interpretations to be considered. They have a joint probability $p_{1 \ldots m}\left(\omega_{1}, \ldots, \omega_{m}\right)$, which has as marginal probabilities $p_{i}\left(\omega_{i}\right), i=1, \ldots, m$. This consideration leads then to the combined hint

$\mathcal{H}_{1} \oplus \ldots \oplus \mathcal{H}_{m}=\left(\Omega_{1} \times \ldots \times \Omega_{m}, P_{1 \ldots m}, \Gamma, \Theta\right)$

In many cases one may assume that the interpretations of the distinct hints are stochastically independent, such that $P_{1 \ldots m}$ is the product measure on $\Omega_{1} \times \ldots \times \Omega_{m}$. For this case of independent hints or sources of information, $(10)$ is called Dempster's rule of combination. The combined interpretations $\left(\omega_{1}, \ldots, \omega_{m}\right)$ for which $\Gamma_{1}\left(\omega_{1}\right) \cap \ldots \cap \Gamma_{m}\left(\omega_{m}\right)$ is empty are called contradictory. If all combined interpretations are contradictory, the hints are not combinable, they are in complete contradiction.

It is possible that the subsets $\Gamma(\omega)$ are all identical and equal to a subset $B$ of $\Theta$ for all interpretations $\omega$. This says simply that the correct answer is for sure in the subset $B$. Such a hint is called deterministic and is simply denoted by $B$. If $\mathcal{H}$ is any other hint relative to the same question, then $\mathcal{H} \oplus B$ can be formed. This is called conditioning of the hint $\mathcal{H}$. The following theorem holds:

Theorem 2.2 (see for example Shafer, 1976 a, Kohlas, Monney, 1995). Let sp, sp(.|B) and $p l, p l(. \mid B)$ denote the support and plausibility functions of $\mathcal{H}$ and $\mathcal{H} \oplus B$ respectively. Then

$s p(H \mid B)=\left(s p\left(H \cup B^{c}\right)-s p\left(B^{c}\right)\right) /\left(1-s p\left(B^{c}\right)\right.$,

$p l(H \mid B)=p l(H \cap B) / p l(B)$. 
This is called Dempster's rule of conditioning. In particular, it is possible that $B=$ $\Theta$. Then the deterministic hint is called vacuous, it carries no information whatsoever concerning the question considered. For a vacuous hint $s p(H)=0$ for all $H \neq \Theta$ and $p l(H)=1$ for all $H \neq \emptyset$. This is a perfect representation of complete ignorance. Clearly we have $\mathcal{H} \oplus \Theta=\mathcal{H}$.

If for all $\omega$ the sets $\Gamma(\omega)$ are singletons, then the hint is called precise or Bayesian (by Shafer, 1976 a). It is then essentially a random variable. In this case $s p=p l$ and $s p$ becomes in fact a probability measure on $\Theta$ and Dempster's rule of conditioning reduces to the usual definition of conditional probability. This is generalized in the following theorem.

Theorem 2.3 (see for example Shafer, 1976 a, Kohlas, Monney, 1995). Let $\mathcal{H}_{1}$ be a precise hint, $\mathcal{H}_{2}$ an arbitrary hint. Then $\mathcal{H}=\mathcal{H}_{1} \oplus \mathcal{H}_{2}$ is also a precise hint. Denote by $p, p_{1}$ the probabilities induced by $\mathcal{H}$ and $\mathcal{H}_{1}$, and by $\mathrm{l}_{2}$ the plausibility function relative to $\mathcal{H}_{2}$. Then, for all $\theta \in \Theta$,

$p(\{\theta\})=k p_{1}(\{\theta\}) p l(\{\theta\}), \quad k^{-1}=\sum_{\theta \in \Theta} p_{1}(\{\theta\}) p l(\{\theta\})$.

This shows that little information is needed in this case from the second hint $\mathcal{H}_{2}$. The plausibilities of the singletons are sufficient, in fact it is even sufficient to know only the relative values of these plausibilities. This is a quite remarkable result, which permits to link evidence theory with the usual Bayesian analysis, a subject which cannot be pursued here.

\section{APPLICATIONS OF EVIDENCE THEORY}

Do the structures introduced above correspond to anything interesting in our surrounding world? Does evidence theory have interesting, nontrivial and convincing applications? Misunderstandings of evidence theory led to disappointing results and as a consequence to a rejection of the theory by some authors. Nevertheless, there are today some domains where evidence theory begins to emerge as a natural approach which gives convincing results. Among these fields are statistical inference, diagnostics, risk analysis and decision analysis. These applications will be surveyed briefly in this section. But in addition to these applications a few others have to be mentioned. At the Stanford Research Institute International, evidence theory was studied very early from an application point of view, see Lowrance et al. 1986, Lowrance, 1988. This institute developed also a Lisp based general system (a shell) for evidential reasoning. Shafer himself applied evidence theory to auditing (Shafer, Srivastava, 1990). Other applications concern image processing (Wesley, 1986; Provan, 1990 b; Lohmann, 1991), geometric and temporal reasoning (Kohlas, Monney, 1991; Monney, $1991 \mathrm{a}$ and b), including project scheduling and financial modelling (Kohlas, 1989). Evidence theory was of course considered as a general approach to uncertainty management in expert systems or knowledge bases. A recent architecture of knowledge bases incorporating evidence theory has been developed by Saffiotti (1991 a and $\mathrm{b}$ ); he addresses in particular the important problem of using an appropriate language like first order logic as a base for the automatic construction of a propositional or extended mathematical model of evidence. 


\subsection{Statistical Inference from an Evidential Point of View}

Dempster's motivation to introduce multivalued mappings and the related lower and upper probabilities was statistical inference (Dempster, 1966, 1967, 1968, 1990). The goal of this new approach was to generalize Bayesian inference and also to reconcile the latter with Fisher's fiducial probabilities. Dempster was able to derive lower and upper probabilities on the unknown parameters to be studied by statistical inference by specifying a multivalued mapping and without using an a priori distribution on the parameter space. If an a priori distribution is known, then Dempster's method can incorporate it and leads to the classical Bayesian approach, but his method works also, if the a priori information about the parameter is less specific than a distribution, if it is only described by a belief function. It unifies and generalizes both Bayesian and Fisher statistics.

This approach will be sketched here in a way proposed by Kohlas (1992), which shows that the lower and upper probabilities of Dempster can in fact be seen as degrees of support and plausibility of hypotheses about the unknown parameter derived from observations and a process model specifying how observations are generated. Let $\Pi$ be the set of possible values of an unknown parameter $\pi$. Inference about this parameter is made by means of an observation process which yields an observation $x$ in some obervation space $X$. It is assumed that the actual observation $x$ depends only on the unknown parameter $\pi$ and a further unknown chance element $\omega$ which belongs to a known probability space $(\Omega, \mathcal{A}, P)$. This is expressed by a known function

$x=f(\pi, \omega)$

Equation (14) is called a process model of observation. Now, if the experiment is carried out, then an observation $x \in X$ becomes available. Given such an observation $x$ and the process model (14), what can be said about the unknown parameter $\pi$ ?

Define $\Gamma_{x}(\omega)=\{\pi \in \Pi: x=f(\pi, \omega)\}$, the set of all possible parameter values if $x$ is the given observation, and $\omega$ the random element which generated the observation. Then $\mathcal{H}_{x}=\left(\Omega, \mathcal{A}, P, \Gamma_{x}, \Pi\right)$ is a hint about the unknown parameter value $\pi$. Hence, if $H \subseteq \Pi$ is any hypothesis about the unknown parameter, then $u_{x}(H)=\left\{\omega \in \Omega: \emptyset \neq \Gamma_{x}(\omega) \subseteq H\right\}$ represents all random elements $\omega$ for which the hypothesis is necessarily true and $v_{x}(H)=$ $\left\{\omega \in \Omega: \Gamma_{x}(\omega) \cap H \neq \emptyset\right\}$ the random elements for which the hypothesis is possibly true. Thus, $s p_{x}(H)=P\left(u_{x}(H)\right) / P\left(v_{x}(\Pi)\right)$ and $p l_{x}(H)=P\left(v_{x}(H)\right) / P\left(v_{x}(\Pi)\right)$ are the degrees of support and plausibility of $H$ given the observation $x$ (assuming that $u_{x}(H), v_{x}(H)$ are measurable). Note that $\Gamma_{x}(\omega)$ may well be empty. But such an $\omega$ cannot possibly have generated the observation $x$, because there is no parameter $\pi$ which in conjunction with $\omega$ generates $x$. Thus, the conditioning in $s p_{x}$ and $p l_{x}$ is well founded. Application of this approach is possible for so called measurement or also structural models (Frazer, 1968; Kohlas, 1992). But also sampling problems may be formulated and analyzed this way (Dempster, 1967 b, 1968 a and b, 1990; Shafer, 1982 a; Kohlas, 1992). Finally, the problem of filtering stochastic dynamic systems may be approached from this point of view (Kohlas, 1991).

A further reference for the application of belief functions in statistical inference is given by Wasserman (1990); this paper takes however not the evidential point of view, but uses belief functions as a means to describe convex sets of a priori probability measures for a 
Bayesian analysis, In the cited paper, other references to similar uses of lower and upper probability in statistical inference may be found.

\subsection{Diagnostics and Risk Analysis}

Diagnostics is the task to infer explanations for a set of observations. In many cases these observations are symptoms of diseases or faults of a medical or technical system. The explanations furnish then the possible diseases causing the observed facts. Symptoms can be considered as evidence for certain possible diseases and evidence theory is therefore an obvious approach to diagnostics.

An early example of a diagnostic expert system was MYCIN. The authors of this system already noted that symptoms are not infallible evidence but may have exceptions. Also they stated that symptoms are evidences which bear on sets of hypotheses rather than on individual hypotheses. They concluded that probability theory was not an appropriate tool for their purposes and they developed their own ad hoc uncertainty calculus. Later, it became clear that evidence theory would have been well adapted to the structure of MYCIN (Gordon, Shortliffe, 1985, Shafer, Logan, 1987). Applications of evidence theory to diagnosis have been discussed by Smets $(1979,1981)$. GERTIS is a newer expert system which is of a similar type (Yen, 1989), although it mixes evidential theory and Bayesian analysis.

In the mean time, a quite different architecture of diagnostic systems emerged, the model based inference systems. Here a model of the properly functioning system serves as a basis to predict output values from given input values. Differences between these predicted values and the observed ones are then used to derive possible diagnoses (a fundamental paper in this domain of logic based diagnosis is Reiter, 1987). These approaches are essentially symbolic and provide no means to rank the possible diagnoses according to their likelihood or to judge the likelihood that a particular component of a system is faulty. It will be explained how these models can be extended in a very natural way by evidence theory to include likelihood measures. Some of these models may not only be used for diagnostic purposes, but for predictive risk analysis as well.

(i) Model Based Diagnostics. A model of a properly functioning system can often be described by a set of variables and relations between them. Let $M=\{1, \ldots, m\}$ be an index set, $X_{i}$ with $i \in M$ a set of variables taking values in the frames $\Theta_{i}$. Let $R$ be a family of subsets of $M$ and for every $J \in R$ let $R_{J} \subseteq \prod_{i \in J} \Theta_{i}=\Theta_{J}$ be a given relation between the variables $X_{i}$ for $i \in J$. These relations correspond to physical devices which realize those relations as input-output relations (where some variables are considered as inputs and the others as outputs). As an example, the $X_{i}$ may be integer variables, taking values in $\mathcal{N}$, and the relations are arithmetic relations like additions $X_{i}+X_{j}=X_{k}$ or multiplications $X_{i} \times X_{j}=X_{k}$. Or else the $X_{i}$ are Boolean variables with values $\{0,1\}$ and the relations are Boolean relations as they arise for example in digital circuits.

If the values of some variables are observed, then such a model permits to test whether the observations are consistent with the assumed relations between the variables. If this is not the case, then some relations must be violated and the corresponding devices must be faulty.

Now fault models of the components can be introduced. Let $\Omega_{J}=\left\{\omega_{0}, \omega_{1}, \ldots, \omega_{s}\right\}$ denote a set of different mutually exclusive working modes of the component associated 
to the relation $J \subseteq M$. It is supposed that the probabilities $p_{J}\left(\omega_{i}\right)$ of these different modes are known. $\omega_{0}$ is assumed to correspond to a properly functioning component, whereas $\omega_{1}$ to $\omega_{s}$ represent different faulty modes. To any mode $\omega_{i}$ corresponds a relation $\Gamma_{J}\left(\omega_{i}\right) \subseteq \Theta_{J} ; \Gamma_{J}\left(\omega_{0}\right)=R_{J}$ is the above relation of a properly functioning device. An and-gate as used in a digital circuit may for example have two fault modes, one where the output is $\mathbf{0}$ for every input configuration and one where the output is entirely unpredictable for each input. The later mode corresponds to the vacuous relation $\{0,1\} \times\{0,1\} \times$ $\{0,1\}$, all combinations of input and output are possible. This associates clearly a hint $\left(\Omega_{J}, p_{J}, \Gamma_{J}, \Theta_{J}\right)$ to every component in the system. The observation of certain variables $X_{i}$ provides further deterministic and precise hints relative to $\Theta_{i}$.

These hints refer to different but related frames. This situation differs from those considered so far and especially in section 2, where hints refered always to the same frame of discernment. The situation encountered here is however typical for most applications of evidence theory. Dempster's rule for the combination of hints can be extended to such situations as will be explained in section 4 . Hence these hints may be combined to obtain degrees of support and plausibility for different diagnoses, that is for hypotheses about the possible faulty configurations of modes which may explain the observed values of variables. The computational methods for this analysis are described in section 4 . This shows that a well known model in diagnosis theory can very naturally be extended using evidence theory so as to incorporate information about the likelihood of possible diagnoses. This approach has been described in Kohlas, Monney, Haenni (1995).

Such systems may also often be conveniently formulated in the framework of logic with uncertain arguments and related to truth maintenance systems (Provan, 1988). A further application of the model introduced above is to the detection of bad data in measurement models (Kohlas, 1992).

(ii) Risk Analysis. Boolean systems as described above arise not only in modeling digital circuits, but also in fault or event trees as used for reliability or risk analysis. These structures can be used for diagnostic purposes, if some events are observed exactly in the way described above. They are however also used for predictive studies of risks. Almond (1991, 1992 a) discusses the statistical analysis of failure rates of components, using methods similar as those described in subsection 3.1. This leads to only partially known component models, in the sense that only belief functions for the unknown parameters can be given. This in turn leads to belief functions for the failure of components and thus for the events in the fault tree. This is an example of how evidence theory may be applied in risk analysis.

\subsection{Decision Analysis}

(i) Generalized Decision Principles. Analysis of uncertain perspectives and data is an important activity in decision support. One aspect is the problem of choice. In the standard choice model, a set $A=\left\{a_{1}, \ldots, a_{n}\right\}$ of possible activities and a set $S=\left\{s_{1}, \ldots, s_{n}\right\}$ of possible states of the world is given, and it is assumed that for every activity $a_{i}$ and state $s_{j}$ a utility index $u_{i j}$ is defined which expresses the preferences of the decision maker. Classical decision theory considers two extreme situations relative to the knowledge of the state of the world. Either there is full ignorance about the state; in this case decision 
principles like the Hurwicz principle, Laplace's principle of insufficient reason or the principle of minimal regret are proposed to select an activity. Or else a probability distribution $p\left(s_{j}\right)$ over the set $S$ is assumed to be given. Then the activity is selected by maximizing the expected value of the utility of an action $a_{i}$

$$
E_{i}\left(u_{i j}\right)=\sum_{j=1}^{n} u_{i j} p\left(s_{j}\right) .
$$

In our terminology, the first case corresponds to a vacuous hint about the state of the world and the second to a precise hint about the state. So it is natural to look at intermediate cases, where an arbitrary hint $\mathcal{H}=(\Omega, p, \Gamma, S)$ about the state is given. If a fixed interpretation $\omega$ is known to be the correct one, then the true state is known to be in the set $\Gamma(\omega)$. With respect to this set of states we have a decision problem with full ignorance and we may apply any one of the decision principles to evaluate the actions. We may then take the expected value over these evaluations for every interpretation $\omega$ in $\Omega$. Each decision principle leads in this way to a generalized decision principle.

First of all, note that an action $a_{i}$ is dominated by an action $a_{k}$, if for all $\omega \max \left\{u_{i j}\right.$ : $\left.s_{j} \in \Gamma(\omega)\right\} \leq \min \left\{u_{k j}: s_{j} \in \Gamma(\omega)\right\}$. Dominated actions may be eliminated from further considerations.

Laplace' principle of insufficient reason amounts to assume a uniform distribution over the states in $\Gamma(\omega)$ for every $\omega$, taking the expected value of the utilities relative to these uniform distributions and then taking the expected value over $\Omega$ :

$$
\sum_{\omega \in \Omega} p(\omega) \sum\left\{u_{i j} /|\Gamma(\omega)|: s_{j} \in \Gamma(\omega)\right\}, \text { for all } a_{i} \in A \text {. }
$$

This generalized principle of insufficient reason has been proposed by Dubois, Prade (1982), Williams (1982), Smets (1989), and Smets, Kennes (1990).

Hurwicz's principle uses an index of optimism $\alpha$ between 0 and 1 to weigh the pessimistic minimal utility value and the optimistic maximal utility value over the possible states. The generalized Hurwicz' principle considers thus the following index for every action $a_{i}$ :

$$
\sum_{\omega \in \Omega} p(\omega)\left(\alpha \max \left\{u_{i j}: s_{j} \in \Gamma(\omega)\right\}+(1-\alpha) \min \left\{u_{i j}: s_{j} \in \Gamma(\omega)\right\}\right) .
$$

This approach has been proposed by Strat (1990). It can also be seen as a linear interpolation between lower and upper bounds of expectation intervals defined by the hint. Strat shows also how to use this principle in the context of generalized decision trees. For $\alpha=1$ the principle reduces to the generalized maximax principle and for $\alpha=0$ to the generalized maximin principle.

The regret principle computes for every state $s_{j}$ and action $a_{i}$ the regret $r_{i j}=\max \left\{u_{i j}\right.$ : $i=1, \ldots, m\}-u_{i j}$. It applies then the generalized minimax principle to the matrix $\left(r_{i j}\right)$. This procedure has not been yet proposed in the literature, but it is in the line of the above principles.

(ii) Expected Utility Theory. Let us generalize the basic decision model introduced above. Instead of describing the outcome of an action $a_{i}$ and a state $s_{j}$ directly by an numerical 
utility index $u_{i j}$, suppose that the outcome is described by some more basic, possibly nonnumeric element $\theta_{i j}=\theta\left(a_{i}, s_{j}\right) \in \Theta$. The hint $\mathcal{H}$ on the set of possible states $s$ is transformed for each action $a_{i}$ by the function $\theta\left(a_{i}, \cdot\right): S \rightarrow \Theta$ into a hint $\mathcal{H}_{i}$ on $\Theta$. The problem of choice of an action $a_{i}$ reduces then to the choice of a hint on $\Theta$ or to a comparison of hints or belief functions on $\Theta$. Now, a hint on $\Theta$ can be seen as probability distribution over the subsets of $\Theta$. Let $\mathcal{P}_{\Theta}$ denote the set of probability distributions over $2^{\Theta}$, where $\Theta$ is assumed to be finite. Then the following theorem holds:

Theorem 3.1 (see for example Fishburn, 1970, theorem 8.2) Let $\prec$ denote a binary relation on $\mathcal{P}_{\Theta}$. Then there is a real-valued function $u$ (called a utility function) on $2^{\Theta}$ that satisfies

$P \prec Q$ iff $\sum_{B \subseteq \Theta} P(B) u(B)<\sum_{B \subseteq \Theta} Q(B) u(B)$

for all $P, Q$ in $\mathcal{P}_{\Theta}$

if and only if, for all $P, Q, R$ in $\mathcal{P}_{\Theta}$

1. $\prec$ on $\mathcal{P}_{\Theta}$ is a weak order,

2. (Independence). $P \prec Q$ implies $\alpha P+(1-\alpha) R<\alpha Q+(1-\alpha) R$ for all $0<\alpha<1$,

3. (Contuinity). $P \prec Q, Q \prec R$ implies $\alpha P+(1-\alpha) R<Q$ and $Q<\beta P+(1-\beta) R$ for some $\alpha, \beta \in(0,1)$.

Moreover, $u$ is unique up to a positive linear transformation $a u+b$.

Inequality (18) states that under the conditions of the theorem the action can be selected by maximizing an expected utility. This has been noted by Jaffray (1989). (18) may however not be very practical given the large numbers of subsets of $\Theta$ for which utilities $u(B)$ must be determined. Jaffray (1989) adds a further condition to (1) to (3) above in theorem 3.1, which reduces (18) essentially to the generalized Hurwicz principle. Jaffray, Wakker (1992) relate the above result to Savage's sure thing principle and show how a weaker version of this principle leads to the above result. Jaffray (1992 a) then considers this expected utility approach also in a dynamic setting.

(iii) Support for Actions. Another approach, but very much in the spirit of evidence theory, is to look for arguments for or against the hypothesis that a given action is satisfactory (achieves the specified goals), or that it is better than another one, or even that it is the best among a given set of actions. Hints and belief functions about the consequences of an action may be used for this purpose. According to Strat (1990) such a "constructive" approach to decision theory has been proposed by Shafer (1982 b) in an unpublished paper. This approach seems not to have been pursued in the literature (see however Schaller, 1991). It would be close in spirit to the outranking methods of multicriteria analysis (see for example Roy, 1985). It should be emphasized that evidential decision analysis as understood here is different from decision analysis with partially known probabilities. 


\section{COMPUTATIONAL ASPECTS OF EVIDENCE THEORY}

The practical implementation of evidence theory leads to several difficult computational problems. How can the available evidence be best encoded for representation on a computer and what are the corresponding memory requirements? Which are the best algorithms to compute degrees of support and plausibility or to combine pieces of evidence by Dempster's rule and what are their complexities? Are there special structures of evidence which facilitate representation and computation? These are the questions which are briefly addressed in this section. References to computer packages which implement evidence theory will also be given.

In practical applications of evidence theory the reasoning concerns a group of distinct variables $X_{1}, \ldots, X_{m}$, each one with its own domain (or frame of discernment) $\Theta_{i}$. Then arbitrary groupings of variables $\left\{X_{j}: j \in J \subseteq M\right.$ (with $M=\{1, \ldots, m\}$ ) can be considered and such a group of variables has the frame $\Theta_{J}=\prod_{j \in J} \Theta_{j}$, the product space of the frames of the variables in the group. Denote $\Theta_{M}$ by $\Theta$; this is the overall frame of the model to be considered. The available pieces of evidence very often do not concern all variables together, but only some groups of them. Such pieces of evidence concerning a group $J$ of variables may be described by the hints $\mathcal{H}_{i}, i=1, \ldots, r$, relative to the frame $\Theta_{J}$. More precisely, let $J_{i}$ denote the group of variables of the hint $\mathcal{H}_{i}$. As an example; consider model based diagnostics described in section 3.2 (ii) above.

As these different hints refer to different frames, they can not be directly combined by Dempster's rule. A hint $\mathcal{H}=\left(\Omega, p, \Gamma, \Theta_{J}\right)$ relative to the group of variables $J$ can be extended to a group $K \supset J$ simply by replacing the focal sets $\Gamma(\omega)$ by their cylindrical extension $\Gamma(\omega) \uparrow K=\Gamma(\omega) \times \Theta_{K-J}$. The new hint $\mathcal{H} \uparrow K=\left(\Omega, p, \Gamma \uparrow K, \Theta_{K}\right)$ is called the vacuous extension of $\mathcal{H}$ from $J$ to $K$. Then the hints relative to different groups of variables may all be vacuously extended to $M$ and then combined on the common frame $\Theta$

$\mathcal{H}=\left(\mathcal{H}_{1} \uparrow M\right) \oplus \ldots \oplus\left(\mathcal{H}_{r} \uparrow M\right)$

In general, let us define Dempster's rule by $\mathcal{H}_{1} \oplus \mathcal{H}_{2}=\left(\mathcal{H}_{1} \uparrow M\right) \oplus\left(\mathcal{H}_{2} \uparrow M\right)$. A representation of $\mathcal{H}$ by its pieces $\mathcal{H}_{i}$ is clearly advantageous from a memory point of view. Not only has each $\mathcal{H}_{i}$ in general much less focal sets than $\mathcal{H}$, but also their focal sets are generally in a much smaller dimension $\left|J_{i}\right|$ than $|M|$.

It seems however at first sight that this last advantage is lost, when the hints are combined, because all of them must be extended to $M$. This however can be avoided in many cases by the ability to combine hints locally. Trivially, if two hints refer to the same group $J$ of variables, then they may be combined on $\Theta_{J}$ beforehand rather than on $\Theta$. Also if one hint refers to a group $J$, the other to a group $K$ and $J \subseteq K$, then the two hints can be combined on $\Theta_{K}$. If all these combinations are executed beforehand, then a family $\mathcal{J}=\left\{J_{1}, \ldots, J_{r}\right\}$ of incomparable groups of variables remain (i.e. all groups are distinct and none is contained in another one). $\mathcal{J}$ is called a scheme (Thoma, 1991) and it defines a hypergraph (with hyperedges $J_{i}$ ).

Now, even beyond the trivial cases above, local combinations are possible, due to a basic theorem given below. If $\mathcal{H}=\left(\Omega, p, \Gamma, \Theta_{J}\right)$ is a hint relative to a group of variables $J$, and $K$ is a subset of $J$, then $\mathcal{H} \downarrow K=\left(\Omega, p, \Gamma \downarrow K, \Theta_{K}\right)$ is the restriction or projection of $\mathcal{H}$ from $J$ to $K$, if $\Gamma(\omega) \downarrow K$ is the projection of $\Gamma(\omega)$ from $\Theta_{J}$ to $\Theta_{K}$. Contrary to the 
vacuous extension, where information is neither added nor lost (i.e. $(\mathcal{H} \uparrow K) \downarrow J=\mathcal{H}$ if $J \subseteq K)$, projecting hints causes in general a loss of information, that is $(\mathcal{H} \downarrow K) \uparrow J \neq \mathcal{H}$, if $K \subseteq J$. Vacuous extension, followed by projection permit to transport a hint relative to a group of variables $J$ to any other group $K, \mathcal{H} \mid K=(\mathcal{H} \uparrow J \cup K) \downarrow K$. Then the following theorems of local computations hold:

Theorem 4.1 (see for example Shafer et al. 1987). If $\mathcal{H}_{1}$ and $\mathcal{H}_{2}$ are hints relative to the groups of variables $H$ and $K$ respectively and $H \cap K \subseteq J$, then

$\left(\mathcal{H}_{1} \oplus \mathcal{H}_{2}\right) \mid J=\left(\mathcal{H}_{1} \mid J\right) \oplus\left(\mathcal{H}_{2} \mid J\right)$

Theorem 4.2 (Shafer et al. 1987). If $\mathcal{H}$ is a hint relative to a group of variables $H$, and $K, J$ denote groups of variables such that $H \cap K \subseteq J$, then

$\mathcal{H}|K=(\mathcal{H} \mid J)| K$

This last theorem shows that transporting a hint from a group of variables $H$ to another group $K$ can be done simpler by projecting the hint first to $H \cap K$ and then extending it to $K$, rather than first extending it to $H \cup K$ and then projecting it to $K$. Theorem 4.1 shows that in some cases hints may be combined locally on certain smaller frames $\Theta_{J}$ relative to a group of variables $J$ rather than on the overall frame $\Theta$.

If $\mathcal{J}$ is a hypergraph, then it may be possible to arrange its hyperedges $J_{i}$ into a family $\mathcal{E}$ of pairs $\left.\left\{J_{i}, J_{k}\right)\right\}$ such that

1. $(\mathcal{J}, \mathcal{E})$ is a tree,

2. if $J_{i}$ and $J_{k}$ are distinct vertices of the tree $(\mathcal{J}, \mathcal{E})$, then $J_{i} \cap J_{k}$ is contained in every vertex on the unique path from $J_{i}$ to $J_{k}$ in the tree $(\mathcal{J}, \mathcal{E})$.

Such a tree is called a Markov tree (also called a join tree in data base theory, see Maier, 1983). If such a tree exists, then $\mathcal{J}$ is called a hypertree or an acyclic hypergraph. If one now considers any vertex $J$ of a Markov tree $T=(\mathcal{J}, \mathcal{E})$, then it has a set $N(J)$ of neighboring vertices and if node $J$ is eliminated then $T$ decomposes into a set of subtrees $T\left(J_{k}\right)$, each one associated with a vertex $J_{k} \in N(J)$. It can be shown that every subtree of a Markov tree is still a Markov tree. If $T=(\mathcal{J}, \mathcal{E})$ is a Markov tree and $\mathcal{J}$ is a scheme, then there is a hint $\mathcal{H}_{J}$ associated with every vertex $J$ of $T$. Let $\mathcal{H}(T)$ denote $\oplus\left\{\mathcal{H}_{J}: J \in \mathcal{H}\right\}$. Property (2) of a Markov tree permits the application of both theorems 4.1 and 4.2 to give the following result:

Theorem 4.3 (Shafer et al. 1987). If $T$ is a Markov tree and $J$ is a vertex of $T$, then

$$
\mathcal{H}(T) \mid J=\mathcal{H}_{J} \oplus\left[\oplus\left\{\left(\mathcal{H}\left(T\left(J_{k}\right)\right) \mid J_{k}\right) \mid J: J_{k} \in N(J)\right\}\right]
$$

This is a recursive formula for combining hints on Markov trees, which can be used to compute projections of the combined hint to groups of variables in the hypergraph $\mathcal{J}$, using local combinations only.

Theorem 4.3 is a basic result for the computational aspects of evidence theory. In 
fact it is a special case of a much more general abstract framework (Shenoy, Shafer, 1990; Shafer, 1991) which covers such diverse models as nonserial dynamic programming (Bertele, Brioschi, 1972), probabilistic (Bayes or Markov) networks (Lauritzen, Spiegelhalter, 1988), constraint propagation, influence diagrams (Oliver, Smith, 1990) and others.

Sometimes the hypergraph has an evident Markov tree structure. Such is the case for example for models of dynamical processes (Kohlas, 1991). However, it is not always possible to construct a Markov tree for a given hypergraph $\mathcal{J}$. In such a case, the hypergraph must be enlarged by augmenting the hyperedges until a Markov tree can be obtained; this is called constructing a hypertree cover of the hypergraph. Although it is not difficult to find a hypertree cover of a hypergraph, it is difficult to find a good one, that is one with hyperedges of small cardinality. There is a growing literature on this subject; see for example Rose, 1970, Bertele and Brioschi, 1972, Tarjan and Yannakakis, 1984, Kong, 1986, Arnborg et al., 1987, Mellouli, 1987, Zhang, 1988 and Almond, Kong, 1991.

Meanwhile, several software packages based on this framework have been developed. Examples are DELIEF (Zarley et al. 1988), MacEvidence (Hsia, Shenoy, 1989), PULCINELLA (Safiotti, Umkehrer, 1991 and 1992), BELIEF (Almond, 1992 b, c) and TRESBEL $(\mathrm{Xu}, 1992)$.

\section{CONCLUSION AND OUTLOOK}

The theory of evidence presented so far may be put into a larger perspection as a theory of probabilistic argumentation systems (Kohlas, 1995). Argumentation systems are in their most general framework algebraic structures which link hypotheses to supporting arguments. This leads then first to an algebraic or symbolic version of evidence theory. Both theoretically and practically important models of such argumentation systems can be derived from assumption-based reasoning formed in general logics (Besnard, Kohlas, 1994) or in particular in propositional logic (Kohlas, 1993 a, Kohlas, Monney, 1995).

The arguments may in a second step be weighted by their probabilities. Thie permits then to measure by what probabilities hypotheses are supported by the argumentation system. In these extended probabilistic argumentation systems the hypotheses are numerically evaluated by degrees of support. This leads then to a very general theory of evidence. The theory of hints sketched above is but one, although important model of this general theory of evidence.

\section{REFERENCES}

Arnborg, S., Corneil, D.G., Proskurowski, A. (1987) Complexity of Finding Embeddings in a k-Tree. SIAM J. Algebraic and Discrete Methods, 8, 277-84.

Almond, R. (1991) Belief Function Models For Simple Series and Parallel Systems. Tech. Report 207, Department of Statistics, Univ. of Washington.

Almond, R. (1992 a) Models for Incomplete Failure Data. Statistical Sciences. Inc., Seattle, WA.

Almond, R. (1992 b) Using the Belief Package. Harvard Univ., Dept. of Statatistics.

Almond, R. (1992 c) Graphical-Belief: Project Overview. Statistical Sciences, Inc., Seattle, WA. 
Almond, R., Kong, A. (1991) Optimality Issues in Constructing Markov Tree from Graphical Models. Res. Rep. A-3, Harvard Univ., Dept. of Stat.

Bertele, U., Brioschi, F. (1972) Nonserial Dynamic Programming. Academic Press.

Besnard, P., Kohlas, J. (1994) Evidence. Theory Based on General Consequence Relations. Tech. Rep. Institute of Informatics, University of Fribourg. To be published in Int. J. of Foundations of Computer Science, 1995.

Bonissone, P.P., Henrion, M., Kanal, L.N., Lemmer, J.F. (eds.) (1991) Uncertainty in Artificial Intelligence 6. North Holland, Amsterdam.

Bouchon-Meunier, B., Yager, R.R., Zadeh, L.A. (eds.) (1991) Uncertainty in Knowledge Bases. Springer Lecture Notes in Computer Science, No. 521.

Dempster, A. (1967 a) Upper and Lower Probabilities Induced by a Multivalued Mapping. Annals Math. Stat., 38, 325-39.

Dempster, A. (1967 b) Upper and Lower Probability Inferences Based on a Sample from a Finite Univariate Population. Biometrika, 54, 515-28.

Dempster, A. (1968 a) A Generalization of Bayesian Inference. J. Royal Stat. Soc., Series $B, \mathbf{3 0}, 205-47$.

Dempster, A. (1968 b) Upper and Lower Probabilities Generated by a Random Closed Interval. Annals Math. Stat., 39, 957-66.

Dempster, A. (1990) Bayes, Fisher, and Belief Functions, in Bayesian and Likelihood Methods in Statistics and Econometrics. (eds. S. Geisser, J.S. Hodges, S.J. Press, A. Zellner)

Dubois, D., Prade, H. (1982) On Several Representations of an Uncertain Body of Evidence in Fuzzy Information and Decision Processes (eds. M.M. Gupta, E. Sanchez) North Holland, Amsterdam, 167-81.

Fishburn, P.C. (1970) Utility Theory for Decision Making. Wiley, New York.

Frazer, D.A.S. (1968) The Structure of Inference. Wiley, New York.

Fedrizzi, M., Kacprzyk, J., Yager, R.R. (eds.) (1993) Advances in the Dempster-Shafer Theory of Evidence. Wiley.

Gordon, J., Shortliffe, E.H. (1985) A Method for Managing Evidential Reasoning in a Hierarchical Hypothesis Space. Artificial Intelligence, 26, 323-57.

Hajek, P., Havranek, T., Jirousek, R. (1992) Uncertain Information Processing in Expert Systems. CRC Press, London.

Hsia, Y., Shenoy, P.P. (1989) Mac Evidence: A Visual Evidential Language for Knowledge Based Systems. Working Paper No. 211, Univ. of Kansas, School of Business.

Jaffray, J.Y. (1989) Linear Utility Theory for Belief Functions. Operations Research Letter, 8, 107-12.

Jaffray, J.Y. (1992) Dynamic Decision Making with Belief Functions. Cahiers de Recherche en Economie, Mathématiques et Applications, Univ. de Paris I, No. 92-15.

Jaffray, J.Y., Wakker, P. (1992) Decision Making with Belief Functions: Compatibility and Incompatibility with the Sure-Thing Principle. Unpubl. Paper, Univ. de Paris VI, LID Paris, France, University of Nijmegen, NICI Nijmegen, The Netherlands.

Kohlas, J. (1989) Modeling Uncertainty with Belief Functions in Numerical Models. European Journal of Operational Research, 40,377-88.

Kohlas, J. (1991) Describing Uncertainty in Dynamical Systems by Uncertain Restrictions, in Modeling, Estimation and Control of Systems with Uncertainty (eds. G.B. Di Masi et al.), Birkhäuser. 
Kohlas, J. (1992) Evidential Reasoning About Parametric Models. Inst. for Automation and Op. Res., University of Fribourg, Switzerland, Tech. Rep. No. 194.

Kohlas, J. (1993 a) Symbolic Evidence, Arguments, Supports and Valuation Networks, in Symbolic an Quantitative Approaches to Reasoning and Uncertainty (eds. M. CLARKE et al.), Springer.

Kohlas, J. (1993 b) Support and Plausibility Functions Induced by Filter-Valued Mappings. Int. J. Gen. Sys., 21, 343-63.

Kohlas, J. (1995) Mathematical Foundations of Evidence Theory, in Mathematical Models for Handling Partial Knowledge in Artificial Intelligence (eds. G. Coletti et al.) Plenum Publ. Corp.

Kohlas, J., Monney, P.A. (1991) Propagating Belief Functions Through Constraint Systems. Int. J. Approximate Reasoning, 5, 433-61. (a shorter version is published in Bouchon et al., 1991, 50-57).

Kohlas, J., Monney, P.A. (1993) Probabilistic Assumption-Based Reasoning. Inst. for Automation and Op. Res., University of Fribourg, Switzerland, Tech. Rep. No. 208.

Kohlas, J., Monney, P.A. (1994 a) Theory of Evidence - A Survey of is Mathematical Foundations, Applications and Computational Aspects. ZOR, Math. Methods of O.R., 39, 35-68.

Kohlas, J., Monney, P.A. (1994 b) Representation of Evidence by Hints, in Advances in the Dempster-Shafer Theory of Evidence (eds. J. Yager et al.), John Wiley, New York.

Kohlas, J., Monney, P.A. (1995) A Mathematical Theory of Hints. Lecture Notes in Economics and Mathematical Systems, Volume 425, Springer-Verlag.

Kohlas, J., Monney, P.A., Haenni, R. (1995) Model-Based Diagnostics Using Hints. To appear in: Springer Lecture Notes in Computer Science.

Kong, A. (1986) Multivariate Belief Functions and Graphical Models. Doct. diss. Harvard Univ., Dept. of Stat.

Kruse, R., Schwecke E., Heinsohn J. (1991) Uncertainty and Vagueness in KnowledgeBased Systems. Springer.

Kruse, R.,; Siegel, P. (eds.) (1991) Symbolic and Quantitative Approaches to Uncertainty. Springer Lecture Notes in Computer Science No. 548.

Lauritzen, S.L., Spiegelhalter, D.J. (1988) Local Computations with Probabilities on Graphical Structures and their Application to Expert Systems. J. Royal Stat. Soc., Series B, 50, 157-224.

Lohmann, G. (1991) An Evidential Reasoning Approach to the Classification of Satellite Images. In Kruse, R., Siegel, P. (1991): 227-31.

Lowrance, J.D. (1988) Automated Argument Construction. J. Stat. Planning Inference, 20, 369-87.

Lowrance, J.D., Garvey, T.D., Strat, T.M. (1986) A Framework for Evidential Reasoning Systems, AAAI-86, 869-903.

Maier, D. (1983) The Theory of Relational Data Bases. Computer Science Press.

Matheron, G. (1975) Random Sets and Integral Geometry. Wiley, New York.

Mellouli, K. (1987) On the Propagation of Beliefs in Networks Using the Dempster-Shafer Theory of Evidence. Doct. Diss., Univ. of Kansas, School of Business.

Monney, P.A. (1991 a) Le raisonnement temporel et géométrique sous incertitude. Doctoral Thesis, Math. Institute, Univ. Fribourg, Switzerland. 
Monney, P.A. (1991 b) Planar Geometric Reasoning With the Theory of Hints, in Computational Geometry. Methods, Algorithms and Applications. (eds. H. Bieri, H. Noltemeier), Lecture Notes in Computer Science, vol. 553, p. 141-59.

Oliver, R.M., Smith, J.Q. (eds.) (1990) Influence Diagrams, Belief Nets and Decision Analysis. Wiley

Provan, G.M. (1988) Solving Diagnostic Problems Using Extended Truth Maintenance Systems. Proc. European Conf on Art. Int., 547-52.

Provan, G.M. (1990 a) A Logic-Based Analysis of Dempster-Shafer Theory. Int. J. Approximate Reasoning, 4, 451-95.

Provan, G.M. (1990 b) The Application of Dempster-Shafer Theory to a Logic-Based Visual Recognition System, in M. Henrion et al. (1990).

Reiter, R. (1987) A Theory of Diagnosis from First Principles. Artificial Intelligence, 32, $57-95$.

Rose, D.J. (1970) Triangulated Graphs and the Elimination Process. J. Math. Anal. and Appl., 32, 597-609.

Roy, B. (1985) Méthodologie Multicritère d'Aide à la Décision. Editions Economica, Paris.

Saffiotti, A. (1991 a) Using Dempster-Shafer Theory in Knowledge Representation, in Bonissone et al. (1991).

Saffiotti, A. (1991 b) A Hybrid Belief System for Doubtful Agents, in Bouchon et. al. (1991).

Saffiotti, A., Umkehrer, E. (1991) PULCINELLA: A General Tool for Propagating Uncertainty in Valuation Networks. Proc. 7-th Conf on Uncertainty in AI, Los Angeles Ca.

Schaller, J.P. (1991) Multiple Criteria Decision Aid Under Incomplete Information. Doctoral Thesis, Economic Sciences Faculty, Univ. of Fribourg, Switzerland.

Shafer, G. (1976 a) A Mathematical Theory of Evidence. Princeton University Press.

Shafer, G. (1976 b) A Theory of Statistical Evidence, in Foundations of Probability Theory, Statistical Inference, and Statistical Theories of Science. (eds. Harper, Hooker), Vol. II, Reidel, Dortrecht.

Shafer, G. (1979) Allocations of Probability. Annals of Prob., 7, 827-39.

Shafer, G. (1982 a) Belief Functions and Parametric Models. J. Royal Statist. Soc. B, 44, $322-52$.

Shafer, G. (1982 b) Constructive Decision Theory. Univ. of Kansas Dept. of Math. Working Paper.

Shafer, G. (1991) An Axiomatic Study of Computations in Hypertrees. Working Paper 232, Business School, Univ. of Kansas.

Shafer, G., Logan, R. (1987) Implementing Dempster's Rule for Hierarchical Evidence. Artificial Intelligence, 33, 271-89.

Shafer, G., Pearl, J. (eds.) (1990) Readings in Uncertain Reasoning. Morgan-Kaufman Publ. San Mateo, Cal.

Shafer, G., Shenoy, P.P., Mellouli, K. (1987) Propagating Belief Functions in Qualitative Markov Trees. Int. J. Approximate Reasoning, 1, 349-400.

Shafer, G., Srivastava, R.P. (1990) The Bayesian and Belief Function Formalisms: A General Perspective for Auditing, Auditing. J. Pract. Theory, 9 (Suppl.).

Shenoy, P.P., Shafer, G. (1990) Axioms for Probability and Belief-Function Propagation, in Shafer, Pearl, 1990. 
Smets, P. (1979) Modèle Quantitatif du Diagnostic Médical. Bulletin de l'Académie de Médecine de Belgique, 134, 330-343.

Smets, P. (1981) Medical Diagnosis: Fuzzy Sets and Degrees of Belief. Fuzzy Sets and Systems, 5, 259-66.

Smets, P. (1988) Belief Functions, in Non-Standard Logics for Automated Reasoning (eds. P. Smets), Academic Press, London.

Smets, P. (1989) Constructing the Pignistic Probability Function in a Context of Uncertainty. Proc. of the Fifth Workshop on Uncertainty in AI, Windsor, Canada, 319-326. Also in Henrion et al. (1990).

Smets, P., Kennes, R. (1990) The Transferable Belief Model. IRIDIA Technical Report TR/IRIDIA/90-14.2, Bruxelles.

Strat, T.M. (1990) Decision Analysis Using Belief Functions. Int. J. Approximate Reasoning, 4, 391-418.

Tarjan, R.E., Yannakakis, M. (1984) Simple Linear Time Algorithms to Test Chordality of Graphs, Test Acyclicity of Hypergraphs, and Selectively Reduce Acyclic Hypergraphs. SIAM J. Computing, 13, 566-79.

Thoma, H.M. (1991) Belief Function Computations, in I.R. Goodman et al. (eds.) (1991). Wasserman, L.A. (1990 a) Prior Envelopes Based on Belief Functions. Annals of Stat., 18, 454-64.

Wasserman, L.A. (1990 b) Belief Functions and Statistical Inference. Can. J. Stat., 18, 183-96.

Williams, P.M. (1982) Discussion of Shafer (1982 a), J. Royal Statist. Soc. B, 44, 342.

Wesley, L.P. (1986) Evidential Knowledge-Based Computer Vision. Optical Engineering, 25, 363-79.

Xu, H. (1992) An Efficient Tool for Reasoning with Belief Functions. Proc. IPMU'92, 65-8.

Yen, J. (1989) GERTIS: A Dempster-Shafer Approach to Diagnosing Hierarchical Hypotheses. Communications ACM, 32, 573-85.

Zarley, D., Hsia, Y.T., Shafer, G. (1988): Evidential Reasoning Using DELIEF, Proc. Nat. Conf, A.I., 1988; Reprinted in Shafer, Pearl, 1990, 619-23.

Zhang, L. (1988): Studies on Finding Hypertree Covers for Hypergraphs. Working Paper No. 198, Univ. of Kansas, School of Business. 